\title{
Molecular Self-Assembly of Enantiopure Heptahelicene-2-Carboxylic Acid on Calcite (10 $\overline{1} 4)$
}

\author{
Christopher M. Hauke, ${ }^{\dagger, \S}$ Philipp Rahe, ${ }^{\dagger}$ Markus Nimmrich, ${ }^{\dagger}$ Jens Schütte, ${ }^{\dagger, \|}$ Markus Kittelmann, ${ }^{\dagger}$ \\ Irena G. Stará,*,‡ Ivo Starý, Jiř́ Rybáček, ${ }^{\ddagger}$ and Angelika Kühnle*, \\ ${ }^{\dagger}$ Institute of Physical Chemistry, Department of Chemistry, Johannes Gutenberg-University Mainz, Jakob-Welder-Weg 11, 55099 \\ Mainz, Germany \\ ${ }^{\ddagger}$ Institute of Organic Chemistry and Biochemistry, Academy of Sciences of the Czech Republic, v.v.i., Flemingovo nám. 2,16610 \\ Prague 6, Czech Republic \\ ${ }^{\S}$ Graduate School of Excellence Materials Science in Mainz, Staudinger Weg 9, D-55128 Mainz, Germany
}

\begin{abstract}
Chirality can have a decisive influence on the molecular structure formation upon self-assembly on surfaces. In this paper, we study the structures formed by enantiopure $(M)$-heptahelicene-2-carboxylic acid $((M)-[7] \mathrm{HCA})$ on the calcite $(10 \overline{1} 4)$ cleavage plane under ultrahigh vacuum conditions. Previous noncontact atomic force microscopy studies have revealed that the racemic mixture of $(M)$-[7]HCA and $(P)$-[7]HCA $(1: 1)$ self-assembles into well-defined molecular double rows that are oriented along the calcite $[01 \overline{1} 0]$ direction. Here, we investigate the enantiopure $(M)$ -

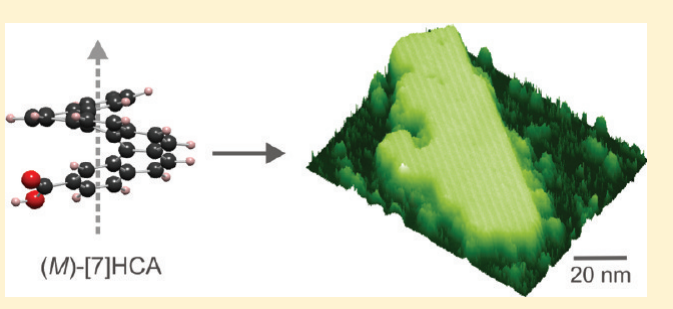

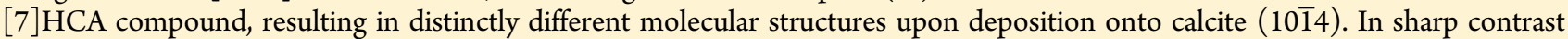
to the racemate, the $(M)$-[7] HCA enantiomer forms molecular islands with a $(2 \times 3)$ superstructure. Comparison of the results presented here for the enantiopure compound with the results previously obtained from the racemate indicates that heterochiral recognition is responsible for the formation of the unidirectional double row structures formed by the racemate.
\end{abstract}

\section{INTRODUCTION}

The influence of chirality on molecular self-assembly on surfaces is of great interest within both fundamental as well as application-oriented fields including, e.g., chiral recognition and enantioselective catalysis. ${ }^{1}$ Ever since Louis Pasteur's famous experiment in 1848 on the manual separation of a racemic mixture, ${ }^{2}$ the spontaneous segregation of a racemate into enantiopure crystallites has attracted great interest. ${ }^{3,4}$ When confined onto a two-dimensional surface, racemic mixtures most commonly form homochiral domains, ${ }^{5,6}$ but heterochiral molecular films having both enantiomers within the unit cell have also been observed. ${ }^{7,8}$ For tartaric acid on $\mathrm{Cu}(110)$, it has been shown that the formation of enantioseparated domains or racemic lattices is coveragedependent. ${ }^{9}$ Besides extended films, chiral recognition has been demonstrated to be decisive for the self-assembly of other structures, e.g., molecular double rows ${ }^{8}$ or dimers. ${ }^{10}$

The detailed influence of chirality at the single molecular level and the substrate templating effect on the resulting mesoscopic or macroscopic expression of chirality is, however, still poorly understood. ${ }^{11,12}$ Moreover, the vast majority of chiral self-assembly investigations have been performed using scanning tunneling microscopy as a direct imaging technique, limiting these studies to metallic surfaces. Recently, we have investigated the self-assembly of racemic heptahelicene-2carboxylic acid ([7]HCA) molecules on the (1014) cleavage plane of calcite using noncontact atomic force microscopy (NC-AFM). ${ }^{13}$ In this work, we have demonstrated the formation of well-defined unidirectional molecular double rows upon adsorption onto the substrate held at room temperature. On the basis of an interplay between NC-AFM results and corresponding density-functional theory (DFT) calculations, we have been able to draw a picture explaining the double row formation. In this model, hydrogen bond formation is responsible for dimerization of [7] HCA molecules, while $\pi-\pi$ interaction results in the unidirectional growth of the molecular rows. This picture is in agreement with a model of heterochiral recognition as the driving force for the double row formation. However, on the basis of the previous results, an unambiguous clarification of this model has not been possible.

Here, we investigate the structure formation of one of the [7]HCA enanitomers, namely, $(M)$-[7]HCA. In sharp contrast to the double rows that have been revealed before for the racemate, the enantiopure compound self-assembles into extended islands composed of rows directed along the [01 $\overline{1} 0]$ direction. This work, thus, elucidates the details of molecular double row formation and indicates that heterochiral recognition is decisive for the unidirectional double rows revealed for the racemic mixture.

The molecule used in this work, namely, the (M)-[7]HCA molecule, is shown in Figure 1a. Seven benzene rings are arranged such as to form a screw-like structure, resulting in two possible helicities of the molecule. For our studies, the heptahelicene was functionalized with a carboxylic group at

Received: October 25, 2011

Revised: December 20, 2011

Published: January 25, 2012 


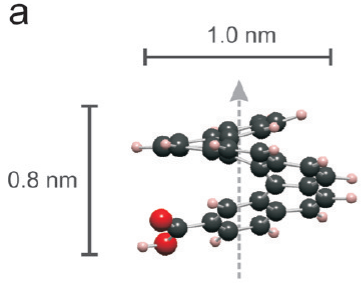

(M)-[7] HCA b

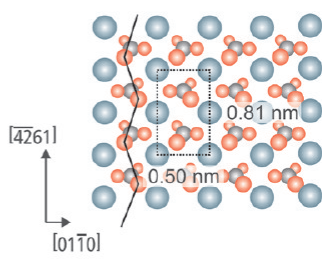

Figure 1. (a) Stick and ball model of the left-handed (M)-[7]HCA molecule. The gray arrow marks the helical axis. (b) Model of the calcite (1014) surface with a rectangular unit cell of $a \times b=0.81 \mathrm{~nm} \times$ $0.50 \mathrm{~nm}$. The two carbonate groups within the unit cell are rotated with respect to each other.

one end of the screw structure. The synthesis of the racemic mixture relies on the $\mathrm{Co}^{I}$-mediated $[2+2+2]$ cycloisomerization of an aromatic triyne to build the helical skeleton. ${ }^{14}$ Separation of the enantiomers was performed by preparative liquid chromatography on a chiral stationary phase column. ${ }^{14}$

The substrate used here is calcite, the most stable polymorph of calcium carbonate. Calcite plays an important role in biomineralization where the motivation is to understand and control its formation process and to fine-tune the resulting materials' properties by interaction with organic molecules. ${ }^{15}$ Calcium carbonate is also used in many industrial products such as paints, paper chemicals, cosmetics, and pharmaceuticals. ${ }^{16}$ Furthermore, adsorption of organic molecules onto calcite has been discussed as a possible origin for the homochirality of life. ${ }^{4,17}$

The surface unit cell of the studied (10 $\overline{1} 4)$ calcite cleavage plane is rectangular with dimensions of $a \times b=0.81 \mathrm{~nm} \times 0.50$ $\mathrm{nm}$. It contains two carbonate groups, which are rotated with respect to the surface normal and with respect to each other. This leads to a characteristic zigzag structure of the topmost oxygen atoms along the [4261] direction, as depicted in Figure 1b. Atomic force microscopy (AFM) and low-energy electron diffraction (LEED) studies have revealed clear signs of a $(2 \times$ 1) reconstruction of the $(10 \overline{1} 4)$ surface. ${ }^{18,19}$ The so-called rowpairing reconstruction, ${ }^{20}$ where every second row along the [4261] direction is imaged brighter than the one before has only been observed in AFM studies so far. Altogether, this leads to a

variety of contrasts that can be observed on the calcite (1014) surface using NC-AFM in ultrahigh vacuum (UHV). ${ }^{21}$

\section{METHODS}

Experiments were performed at room temperature (RT) in a UHV system with a base pressure lower than $1 \times 10^{-10}$ mbar. The system is equipped with an atomic force microscope (VT AFM XA from Omicron, Taunusstein, Germany) and phaselocked loop detector and amplitude controller (easyPLL Plus from Nanosurf, Liestal, Switzerland) for signal demodulation and oscillation excitation. We used n-doped silicon cantilevers (NanoWorld, Neuchâtel, Switzerland) with resonance frequencies of about $300 \mathrm{kHz}$ (type PPP-NCH), excited to oscillation with an amplitude of about $10 \mathrm{~nm}$. Prior to their use, cantilevers were $\mathrm{Ar}^{+}$sputtered at $2 \mathrm{keV}$ for $1 \mathrm{~min}$ to remove contaminants. Depending on the feedback loop settings, either frequency shift or topography images are obtained. The images are displayed such that bright corresponds to highly attractive interaction while dark corresponds to less attractive or even repulsive interactions.

The calcite crystals in use are of the highest available quality from Korth Kristalle GmbH (Altenholz, Germany) and have a rectangular cross section of $2 \times 4 \mathrm{~mm}^{2}$. They are degassed at $475 \mathrm{~K}$ for $2 \mathrm{~h}$ to remove contaminants, cleaved in situ, ${ }^{22}$ and annealed at $475 \mathrm{~K}$ for $2 \mathrm{~h}$ to remove surface charges.

The (M)-[7]HCA molecules are deposited onto the surface under UHV conditions by sublimation from a home-built Knudsen cell at a temperature of about $420 \mathrm{~K}$. During the deposition process, the substrate is kept at room temperature. The samples are transferred into the AFM situated in the same UHV system immediately after deposition.

\section{RESULTS AND DISCUSSION}

First, we discuss the structures formed after depositing the lefthanded $(M)$-[7]HCA molecule onto the calcite (1014) held at RT. A representative image is given in Figure 2a. In this image, island structures are revealed that are oriented along the [01 10$]$ direction of the underlying substrate. The enantiopure islands are, except for their orientation, distributed randomly over the surface at a coverage of $<0.1$ monolayer. Two step edges, running as straight lines from top to bottom, can be seen at the right side of the image. The islands are formed on flat terraces,
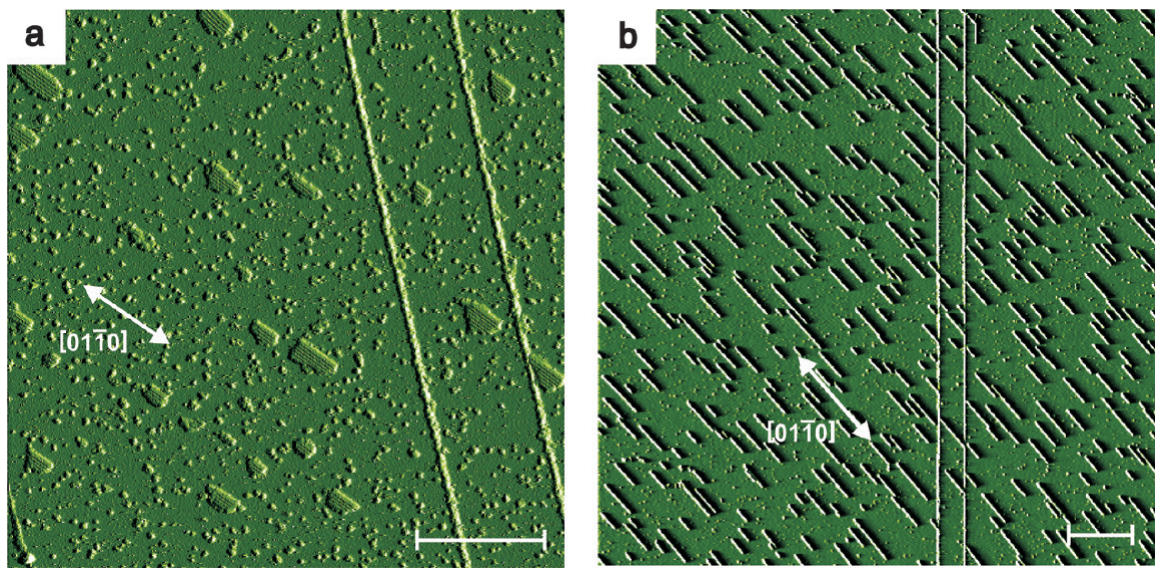

Figure 2. Frequency shift images of enantiopure and racemic [7] HCA on calcite (1014) (scale bars = $100 \mathrm{~nm})$. (a) Enantiopure (M)-[7]HCA islands. (b) Racemic [7]HCA mixture forming unidirectional double rows. Image adapted from ref 13. Both images show calcite step edges, visible as straight lines running from top to bottom. 

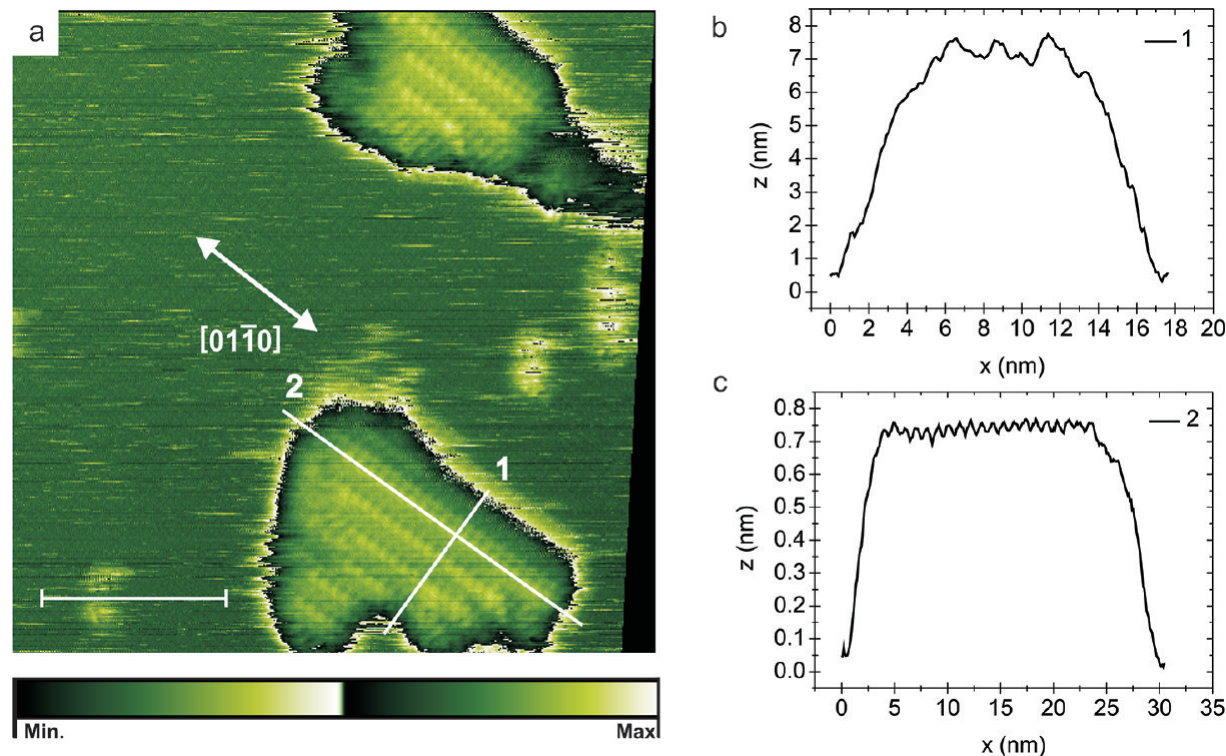

Figure 3. (a) High-resolution topography image of molecular islands formed by $(M)$ - $[7] \mathrm{HCA}$ (scale bar $=15$ nm, image corrected for linear drift). The underlying calcite substrate is resolved simultaneously. A double color scale (see color bar below) is used for data representation in order to enhance visibility. ( $b$ and $c$ ) Height profiles along the calcite [4261] and [01 $\overline{1} 0]$ direction, respectively, as indicated by the drawn lines.

and the step edges do not provide nucleation centers for island growth. Even at increased coverages, no agglomeration at the step edges takes place (not shown). These results obtained for the enantiopure compound differ significantly from the structures that have been observed before for the racemic mixture of [7] HCA. ${ }^{13}$ A typical image showing the structures revealed after deposition of the racemate is reproduced in Figure $2 \mathrm{~b}$. In the previous study using the racemate, we have shown that unidirectional rows are formed aligned along the

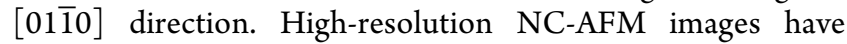
revealed that these structures are composed of molecular double rows. In combination with DFT calculations, we have proposed a model for the double row formation, which is based on dimerization through hydrogen bond formation of the carboxylic groups, while $\pi-\pi$ interaction leads to a binding of the molecular dimers along the row direction. Although we had already speculated that heterochiral recognition might take place in the case of the racemate, our previous results did not allow for an unambiguous identification whether the observed rows were homochiral or heterochiral. This question can now be addressed on the basis of the results obtained in the present study using the enantiopure compound. The fact that racemic and enantiopure [7] HCA form distinctly different structures on the same substrate is a strong indication that the previously observed molecular double rows indeed originate from heterochiral recognition. This will be further corroborated by the detailed investigation of the enantiopure islands presented in the following.

Figure 3a shows a high-resolution image of two $(M)$-[7] HCA islands. Note that the underlying calcite substrate is imaged simultaneously, providing an inherent lateral length calibration. The (M)-[7]HCA islands are imaged with an apparent height of typically $0.7 \mathrm{~nm}$, as shown in the height profiles provided in Figure $3 \mathrm{~b}, \mathrm{c}$. Considering the van der Waals radius of an individual molecule, a height of about $1.15 \mathrm{~nm}$ would be expected for upright standing molecules (i.e., the helical axis being vertical to the surface plane). This discrepancy to the apparent height measured in our study can be readily explained if we assume that the adsorbed molecules' helical axis is tilted relative to the surface plane. This interpretation is in agreement with our previous studies on the racemic mixture. A tilted adsorption geometry was also observed by Ernst et al. for heptahelicene molecules in a saturated monolayer on $\mathrm{Ni}(100) .{ }^{23}$ Already at the scale presented in Figure 2a (image size of $435 \times 435 \mathrm{~nm}^{2}$ ), the $(M)$-[7]HCA islands clearly reveal an inner structure, consisting of parallel rows oriented along the

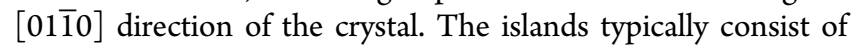
4-6 of these rows. The rows exhibit a repeat distance of $2.3 \pm$ $0.1 \mathrm{~nm}$ along the $[\overline{42} 61]$ direction, as confirmed by the height profile given in Figure $3 \mathrm{~b}$. This distance is about twice as large as the distance one would expect for two neighboring $(M)$ [7]HCA molecules, indicating that two molecules align along this direction with one molecule being imaged brighter than the other. The observed height difference between these two molecules is in the order of $0.02-0.05 \mathrm{~nm}$. Regarding these apparent height differences, we want to stress that the measured variable in NC-AFM is the change in the cantilever's resonance frequency due to the interaction of tip and sample. Use of a feedback loop then allows one to gain topography information from this frequency shift. This means that the measured height represents a plane of equal interaction and might, for example, also be influenced by the shape of the tip or electrostatic forces as well. ${ }^{24}$

Along the molecular rows, a clearly visible corrugation following the calcite $[01 \overline{1} 0]$ direction can be identified, as shown in the height profile in Figure 3c. The corrugation exhibits a periodicity of $1.0 \pm 0.1 \mathrm{~nm}$, which agrees with an assignment of each of these bright spots to a single $(M)$ [7] HCA molecule. Overall, a $(2 \times 3)$ superstructure can be assigned for the $(M)$-[7]HCA islands, as depicted in Figure 4.

A model suggested by the NC-AFM data for the molecules' adsorption geometry is given in Figure 5. We assume that the $(M)$-[7]HCA molecules adsorb with the carboxylic acid moiety pointing toward the calcite surface, where the carbonyl oxygen is expected to bind to a surface calcium atom, whereas the hydroxyl group will bind to an oxygen atom of a carbonate 


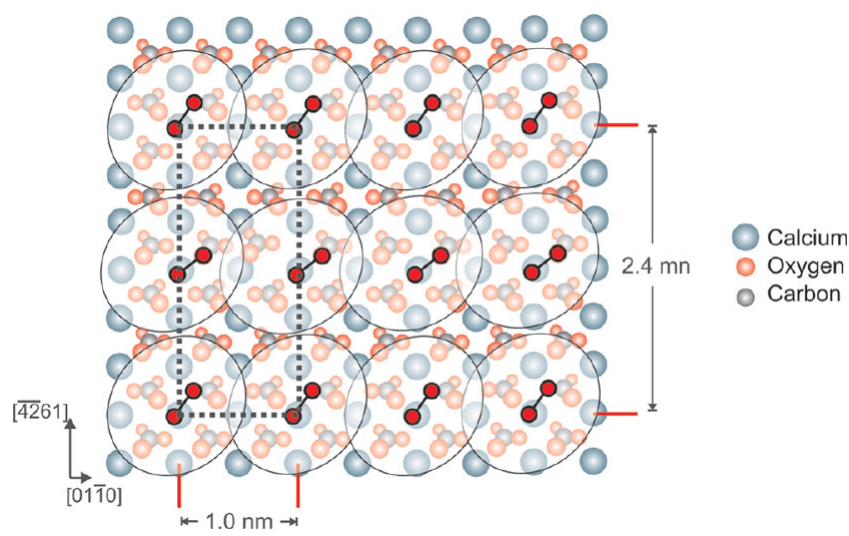

Figure 4. Top view of the calcite $(10 \overline{1} 4)$ surface with adsorbed $(M)$ [7] HCA molecules. The $(M)$-[7]HCA molecules are represented by their van der Waals radii (shaded ellipses) and the carboxylic groups only (linked red circles representing the oxygen atoms). The unit cell of the $(2 \times 3)$ superstructure is marked by the dashed rectangle.
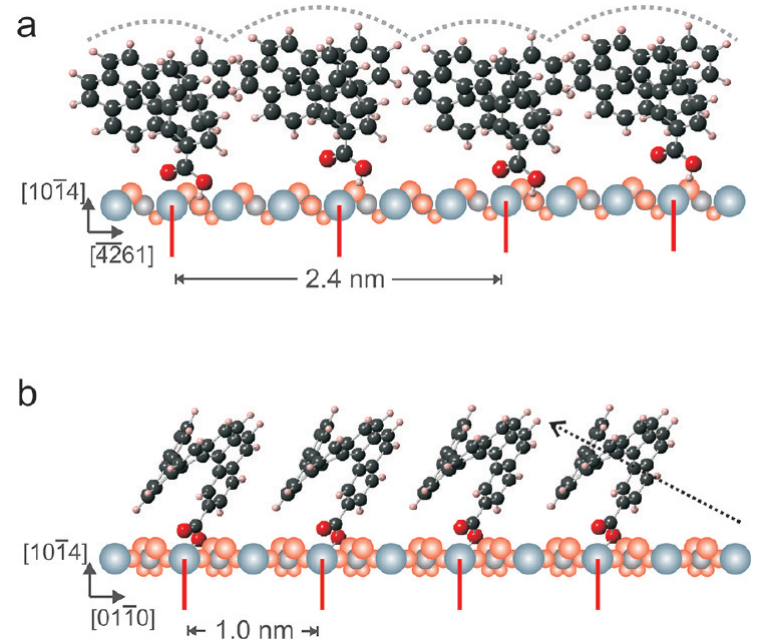

Figure 5. Side views of the calcite (1014) surface with the suggested adsorption geometry of $(M)$-[7]HCA molecules. (a) Along the [4261] direction, the measured repeat distance is $2.4 \mathrm{~nm}$. Binding to different surface carbonate groups leads to a different orientation of the molecules. (b) Along the [01 $\overline{1} 0]$ direction, the measured repeat distance is $1.0 \mathrm{~nm}$, twice the unit cell repeat distance of calcite along this direction. The dashed arrow marks the molecules' helical axis to indicate the tilt.

group. Such a binding behavior has been studied theoretically ${ }^{25}$ and agrees well with the experimentally determined binding of other $-\mathrm{COOH}$ functionalized molecules. ${ }^{26}$ As mentioned above, the two carbonate groups within the unit cell are rotated with respect to each other. This results in a different binding geometry for every second $(M)$-[7]HCA molecule along the $[\overline{42} 61]$ direction. This fact provides a straightforward explanation for the different appearance of the two molecules along this direction: The different binding geometry inevitably results in a different orientation of the molecules with respect to each other, which apparently is propagated to a height difference.

We now continue this discussion by studying the morphology evolution of the formed island structures. Imaging directly after deposition of the molecules (i.e., approximately 1 $\mathrm{h}$ after deposition) already reveals the aforementioned islands, together with a high density of smaller features (not shown here). During the scanning process, the density of the latter decreased, whereas the islands grew in area. We hence assume that most of these smaller features were indeed small $(M)$ [7]HCA units, diffusing freely on the surface until binding to already formed islands. This is similar to what has been revealed for the racemate, where a transient row structure has been observed in images taken approximately $1 \mathrm{~h}$ after deposition, consisting of single rows that transform into the double rows discussed above. ${ }^{13}$ Single rows have also been found for the enantiopure compound shortly after deposition, although their observed density is somewhat less than for the racemate. Assuming the racemic double rows to be of heterochiral composition, the single rows observed in that case are presumably formed by only one of the enantiomers. Over time, the single rows vanish and exclusively molecular double rows remain, which leads to the conclusion that the molecular double rows are energetically favored over molecular single rows. When depositing the (M)-[7]HCA molecules, enantiopure units have no counterpart to form heterochiral structures such as the double rows, and thus, they agglomerate into islands instead. In order to evaluate whether a morphology evolution of the (M)-[7] HCA islands takes place on longer time scales than the usual scanning process, some of the samples were stored under UHV conditions after the initial molecule deposition and imaging. We waited for three days and performed additional imaging after that period. These measurements did not reveal any changes in the morphology of the island structures, indicating that the island structures represent a rather stable configuration at this temperature.

To investigate the effect of higher temperatures on the formed structures, (M)-[7]HCA was deposited on a freshly prepared calcite sample and subsequently heated. Prior to heating, the sample was imaged to ensure that the expected island formation had taken place. Heating up to temperatures of $\sim 360 \mathrm{~K}$ produced no observable change in morphology. However, when increasing the temperature further to $\sim 400 \mathrm{~K}$ and annealing the sample for $1 \mathrm{~h}$, we found that the number of molecular islands had decreased, whereas their individual areas had increased. The average increase in island size is of a factor of 4-6. The inner island structure, however, was not changed compared to the situation before annealing, as can be seen in the image shown in Figure 6. The orientation of these annealed islands remained along the calcite $[01 \overline{1} 0]$ direction, which emphasizes the strong templating effect of the underlying substrate. As before, the step edges did not act as nucleation centers for the $(M)$-[7]HCA islands. Further elevating the annealing temperature to $\sim 415 \mathrm{~K}$ caused the islands to vanish, and we did not observe any other ordered structures. In particular, no molecular double rows were revealed as has been found for the racemic mixture.

\section{CONCLUSIONS}

We report the formation of homochiral (M)-[7] HCA islands on the calcite (10̄14) surface. The substrate has a strong templating effect on the adsorption of the molecules, which is shown by the common orientation of the islands along the

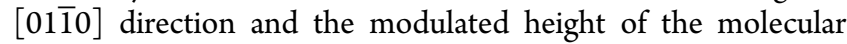
rows along the [4261] direction. This island formation is in sharp contrast to the formation of unidirectional double rows, which has been observed before for the deposition of racemic [7] HCA onto the same surface. Our findings, thus, demonstrate the influence of chirality on the double row 

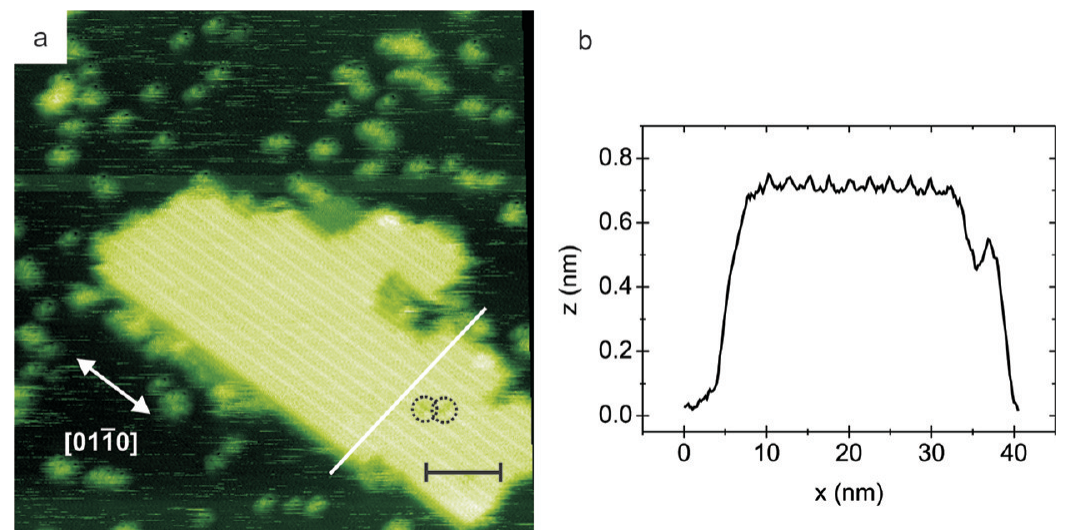

Figure 6. (a) (M)-[7]HCA island after annealing to $\sim 400 \mathrm{~K}$ for $1 \mathrm{~h}$ (scale bar $=25 \mathrm{~nm}$, image corrected for linear drift). The dashed circles show defects on the molecular rows. (b) Height profile along the indicated line. The annealed islands do not show differences in height and periodicity in comparison to the island structures observed at room temperature.

formation and indicate that heterochiral recognition is responsible for row formation of the racemate.

\section{AUTHOR INFORMATION}

\section{Corresponding Author}

*E-mail: kuehnle@uni-mainz.de (A.K.); stara@uochb.cas.cz (I.G.S.). Phone: +4961313923930 (A.K.); +420 220183 315 (I.G.S.). Fax: +4961313953930 (A.K.); +420 220183 133 (I.G.S.).

\section{Present Address}

"Dr. Eberl MBE-Komponenten GmbH, Gutenbergstraße 8, 71263 Weil der Stadt, Germany.

\section{ACKNOWLEDGMENTS}

Financial support from the German Research Foundation (DFG) through the SFB 625 grant TP B17 is gratefully acknowledged. C.M.H. is a recipient of a fellowship through the Excellence Initiative (DFG/GSC 266). This research was supported by the Czech Science Foundation (under Grant No. P207/10/2207) and by the Institute of Organic Chemistry and Biochemistry, Academy of Sciences of the Czech Republic (part of the Research Project Z4 055 0506).

\section{REFERENCES}

(1) Ernst, K.-H. Top. Curr. Chem. 2006, 265, 209-252.

(2) Pasteur, L. Ann. Chim. Phys. 1848, 24, 442-459.

(3) Sheldon, R. A. Chirotechnology; Marcel Dekker Inc.: New York, Basel, 1993.

(4) Addadi, L.; Weiner, S. Nature 2001, 411, 753-755.

(5) Eckhardt, C.; Peachey, N.; Swanson, D.; Takacs, J.; Khan, M.; Gong, X.; Kim, J.-H.; Wang, J.; Uphaus, A. Nature 1993, 362, 614616.

(6) De Feyter, S.; De Schryver, F. C. Chem. Soc. Rev. 2003, 32, 139150.

(7) Fasel, R.; Parschau, M.; Ernst, K.-H. Nature 2006, 439, 449-452.

(8) Böhringer, M.; Schneider, W.-D.; Berndt, R. Angew. Chem., Int. Ed. 2000, 39, 792-795.

(9) Romer, S.; Behzadi, B.; Fasel, R.; Ernst, K.-H. Chem.-Eur. J. 2005, 11, 4149-4154.

(10) Kühnle, A.; Linderoth, T. R.; Hammer, B.; Besenbacher, F. Nature 2002, 415, 891-893.

(11) Mark, A. G.; Forster, M.; Raval, R. ChemPhysChem 2011, 12, $1474-1480$

(12) Yu, M.; Kalashnyk, N.; Xu, W.; Barattin, R.; Benjalal, Y.; Laegsgaard, E.; Stensgaard, I.; Hliwa, M.; Bouju, X.; Gourdon, A.;
Joachim, C.; Besenbacher, F.; Linderoth, T. R. ACS Nano 2010, 4, 4097-4109.

(13) Rahe, P.; Nimmrich, M.; Greuling, A.; Schütte, J.; Stará, I.; Rybáček, J.; Huerta-Angeles, G.; Starý, I.; Rohlfing, M.; Kühnle, A. J. Phys. Chem. C 2010, 114, 1547-1552.

(14) Rybáček, J.; Huerta-Angeles, G.; Kollárovič, A.; Stará, I. G.; Starý, I.; Rahe, P.; Nimmrich, M.; Kühnle, A. Eur. J. Org. Chem. 2011, 853-860.

(15) Addadi, L.; Weiner, S. Angew. Chem., Int. Ed. Engl. 1992, 31, $153-169$

(16) Cölfen, H. Curr. Opin. Colloid Interface Sci. 2003, 8, 23-31.

(17) Orme, C. A.; Noy, A.; Wierzbicki, A.; McBride, M. T.; Grantham, M.; Teng, H. H.; Dove, P. M.; DeYoreo, J. J. Nature 2001, $411,775-779$.

(18) Stipp, S. L.; Hochella, M. F. Geochim. Cosmochim. Acta 1991, 55, $1723-1736$.

(19) Schütte, J.; Rahe, P.; Tröger, L.; Rode, S.; Bechstein, R.; Reichling, M.; Kühnle, A. Langmuir 2010, 26, 8295-8300.

(20) Stipp, S. L. S.; Eggleston, C. M.; Nielsen, B. S. Geochim. Cosmochim. Acta 1994, 58, 3023-3033.

(21) Rahe, P.; Schütte, J.; Kühnle, A. J. Phys.: Condens. Matter 2011, 23.

(22) Tröger, L.; Schütte, J.; Ostendorf, F.; Kühnle, A.; Reichling, M. Rev. Sci. Instrum. 2009, 80, 063703.

(23) Ernst, K.-H.; Neuber, M.; Grunze, M.; Ellerbeck, U. J. Am. Chem. Soc. 2001, 123, 493-495.

(24) Sadewasser, S.; Lux-Steiner, M. C. Phys. Rev. Lett. 2003, 91, 266101.

(25) Duffy, D. M.; Harding, J. H. J. Mater. Chem. 2002, 12, 34193425.

(26) Kittelmann, M.; Rahe, P.; Nimmrich, M.; Hauke, C. M.; Gourdon, A.; Kühnle, A. ACS Nano 2011, 5, 8420-8425. 